Waltershausen, Bodo Sartorius Freiherr von. Paracelsus am Eingang der deut. schen Bildungsgeschichte. Forschungen z. Gesch. d. Philosophie und der Pädagogik, Heft 16, Verlag F. Meiner, Leipzig 1936, 216 S.

- Theophrastus Paracelsus. Entdecker der Chemie. Die Pause, 6. Jahrgang, 9. Heft, Wien 1941.

Wegener, Ilse. Paracelsus. Die Frau, Berlin, Sept. 1941.

Werle, Fritz. Paracelsus. Nova Acta Paracelsia Bd. I. S. 169-181, Birkhäuser Basel 1944.

Zeller, Eberhard. Paracelsus der Beginner eines deutschen Arzttums. Werkstätten der Stadt Halle Burg Giebichenstein (o. J. 1935?)

Zilboorg, Gregory, siehe Temkin, C. L.

Zimmermann, Walter. Der Grund der Arznei ist die Liebe. Paracelsus und die deutsche Arzneimittellehre (illustr.). Die Pause. 6. Jahrg., 9. Heft, Wien 1941.

H. Fischer.

\title{
Buchbesprechungen
}

Vesaliana. A Bio-Bibliography of Andreas Vesalius.. By Harvey Cushing. (Publication No 6 Historical Library, Yale University Library) Pp XXXXVIII $111+230+86$ plates. New York Schumanns 1943. 15.-\$.

Harvey Cushing, der klassische Meister der Neurochirurgie und einer der großzügigsten und tief interessierten Sammler medizinhistorischer Werke hat sich während 40 Jahren seines reichen Lebens mit Vesal beschäftigt. Zwanzig Jahre wandte er daran, um die für europäische Verhältnisse fast unerschwinglich gewordenen Originalien zu seiner Vesal-Bio-Bibliographie zu sammeln, welche ihn bis in die letzten Tage seines Lebens beschäftigte. Leider blieb das kostbare, mit 86 Tafeln geschmückte Werk, welches in neun Kapiteln die Hauptstationen des vesalianischen Lebenswerkes behandelt und in einem letzten, 10. Kapitel die sehr wertvolle Vesal-Bibliographie enthält, Torso. J. F. Foulton hat das Werk seines Freundes zu Ende geführt und damit der Vesal-Forschung einen großen Dienst geleistet. - Eine ausführliche Besprechung des "Vesaliana» aus der Feder J. N. L. B akers findet sich in Nature 153, 694, (1944).

Es darf in diesem Zusammenhang erwähnt werden, daß Harvey Cushings glänzend ausgestattete medizinhistorische Bibliothek, welche Vesal'sche Originalausgaben und die Vertreter der Renaissancemedizin in unerreichter Vollständigkeit beherbergt, seit Jahresfrist in einem Bibliotheksneubau der Yale University (als Legat Cushings) Aufstellung fand. Dorthin wird auch die 
mit so großer Liberalität dem medizinhistorisch Forschenden immer zur Verfügung gestellte Bibliothek unseres unvergeßlichen Arnold C. Klebs, $\dagger$ am 6. März 1943 in Nyon, nach Kriegsende gelangen und durch ihre reichen Bestände an mittelalterlicher und Frührenaissanceliteratur die Sammlung seines Freundes Cushing (wie schon zu Lebzeiten beschlossen) in wertvollster Weise ergänzen. Mit dem Bedauern, daß diese wohl schönste medizinhistorische Bibliothek den Schweizerboden bald verlassen wird, sei die Hoffnung verbunden, es möchte auch bei uns sich jemand finden, welcher mit der gleichen Großzügigkeit und Liberalität sich um die Schaffung einer medizin-historischen Bibliothek verdient machen möchte, durch welche Forscher auf den Gebieten der historischen Medizin, Naturwissenschaft und Technik in den Stand gestellt würden, sich am Heben der so reichen literarischen Schätze unseres Landes, welche in vielen öffentlichen und privaten Bibliotheken der Schweiz ruhen, in ausgiebigerem Maße zu beteiligen, als dies bis heute möglich war. Solange ein gut ausgestattetes medizinhistorisches Zentrum in der Schweiz fehlt, wird auch der medizinhistorische Unterricht, der für den werdenden Arzt so reiche Bildungselemente enthält, nur ein untergeordnetes Dasein fristen können - dies in starkem Gegensatz zu den meisten europäischen Kulturstaaten, welche wie Schweden, Polen, Italien, Frankreich, Holland, Deutschland usw. medizinhistorische Forschung und Unterricht zum Teil in hervorragender Weise geför: dert und zu einem lebendigen Bestandteil der Kulturgeschichte ihres Landes entwickelt haben.

\section{H. Fischer.}

Johann Heinrich Lambert, Schriften zur Perspektive. Herausgegeben und ein. geleitet von Max Steck. 496 S. Dr. Georg Lüttke Verlag. Berlin 1943.

Zu den bedeutendsten Werken des großen Mathematikers J. H. Lambert, der sich selbst als «Mulhusino-Helveticus» bezeichnete und jüngerer Zeitgenosse Eulers war, gehört seine «Freye Perspektive», die 1759 gleichzeitig in deut. scher und französischer Ausgabe in Zürich bei Heidegger erschien. Dieses Werk, das 1774 eine zweite Auflage erfuhr, begründete die moderne Perspektive, und zwar ebenso in geometrischer wie in ästhetischer Richtung. Seit lan. ger Zeit ist das Buch jedoch vergriffen und leider auch in Ostwalds Klassikern der exakten Wissenschaften nicht neu aufgelegt worden. Es bedeutet daher ein großes Verdienst des deutschen Gelehrten, der als Dozent für Mathematik an der Technischen Hochschule und Universität München amtet, eine neue, reich dokumentierte und staatliche Ausgabe veranstaltet zu haben. Ein besonderes Verdienst liegt darin, daß er auch wertvolle, bisher unveröffentlichte Manuskripte Lamberts zur Perspektive anfügte (so die "Anlage zur Perspektive» von 1752, und «Kurzgefaßte Regeln zu perspektivischen Zeichnungen vermittelst eines zu deren Ausübung so wie auch zu geometrischen Zeichnun. 